\title{
Construção de instrumento guia para pesquisa com populações amazônicas a partir das determinações sociais em saúde
}

Construction of a guiding instrument for research with amazon populations based on social determinations in health

Construcción de un instrumento orientador para la investigación con poblaciones amazónicas basado en determinaciones sociales en salud

Recebido: 25/01/2022 | Revisado: 29/01/2022 | Aceito: 30/01/2022 | Publicado: 01/02/2022

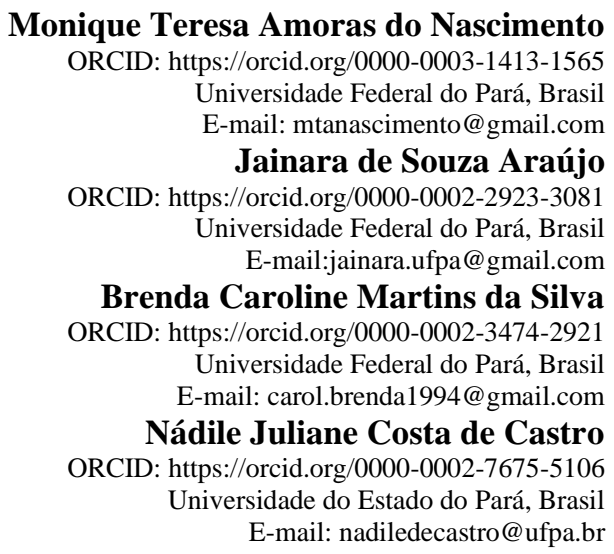

\begin{abstract}
Resumo
Objetivo: Relatar experiência da construção de instrumento guia para pesquisa de campo com populações Amazônicas. Método: Relato de experiência do tipo descritivo realizado a partir do método Blended Learning. Foi realizada entre agosto de 2020 a julho de 2021 por meio de atividades de um Grupo de Estudo e Pesquisa e um projeto de pesquisa da Faculdade de Enfermagem da Universidade Federal do Pará durante o período Remoto Emergencial. Participaram da experiência três discentes e um docente. Resultado: Desenvolveu-se o instrumento-guia por meio do ensino-aprendizagem da saúde das populações em situação de vulnerabilidade no contexto amazônico a partir de treinamento sobre pesquisa na Amazônia, modo de vida de populações ribeirinhas, conceitos sobre questões socioambientais e determinações em saúde e das Políticas Públicas de Saúde. Considerações Finais: A formação de pesquisadores para pesquisa no contexto amazônico deve ser realizada por meio de treinamentos que promovam o pensamento crítico a partir da realidade regional, com instrumentos implementados a partir das políticas públicas de saúde para populações do campo, águas e florestas, assim como da atenção à saúde e dos serviços direcionados às populações ribeirinhas.
\end{abstract}

Palavras-chave: Aprendizagem; Guia; Populações vulneráveis; Determinantes Sociais da saúde; Pensamento.

\begin{abstract}
Objective: To report the experience of building a guide instrument for field research with Amazonian populations. Method: Descriptive experience report based on the Blended Learning method. It was carried out between August 2020 and July 2021 through the activities of a Study and Research Group and a research project at the Faculty of Nursing of the Federal University of Pará during the Remote Emergency period. Three students and one teacher participated in the experiment. Result: The guide instrument was developed through teaching-learning on the health of vulnerable populations in the Amazon context, based on training on research in the Amazon, the way of life of riverside populations, concepts on socio-environmental issues and health determinations and Public Health Policies. Final Considerations: The training of researchers for research in the Amazon context must be carried out through training that promotes critical thinking based on the regional reality, with instruments implemented based on public health policies for populations in the countryside, water and forests, as well as health care and services aimed at riverside populations.
\end{abstract}

Keywords: Learning; Guide; Vulnerable populations; Social determinants of health; Thought. 


\begin{abstract}
Resumen
Objetivo: Reportar la experiencia de construcción de un instrumento guía para la investigación de campo con poblaciones amazónicas. Método: Informe de experiencia descriptivo basado en el método Blended Learning. Se llevó a cabo entre agosto de 2020 y julio de 2021 a través de las actividades de un Grupo de Estudio e Investigación y un proyecto de investigación en la Facultad de Enfermería de la Universidad Federal de Pará durante el período de Emergencia Remota. Tres estudiantes y un profesor participaron en el experimento. Resultado: El instrumento guía fue elaborado a través de la enseñanza-aprendizaje sobre la salud de las poblaciones vulnerables en el contexto amazónico, a partir de capacitaciones sobre investigaciones en la Amazonía, el modo de vida de las poblaciones ribereñas, conceptos sobre cuestiones socioambientales y determinaciones de la salud y público. Políticas de Salud Consideraciones Finales: La formación de investigadores para la investigación en el contexto amazónico debe realizarse a través de una formación que promueva el pensamiento crítico a partir de la realidad regional, con instrumentos implementados a partir de políticas públicas de salud para las poblaciones del campo, el agua y los bosques, así como la atención y servicios de salud dirigidos a las poblaciones ribereñas.
\end{abstract}

Palabras clave: Aprendizaje; Guía; Poblaciones vulnerables; Los determinantes sociales de la salud; Pensó.

\title{
1. Introdução
}

As práticas de ensino-aprendizagem ao longo dos anos têm apontado estratégias que incorporam ações ativas, críticasreflexivas a fim de instrumentalizar os profissionais em formação a partir de modelos inovadores (Sena, et al., 1999; Alves \& Teo, 2020; Riegel, et al., 2021). Em tempos de pandemia a necessidade de mudanças ficou evidente, e o desenvolvimento de práticas pedagógicas inovadoras por meio das ações institucionais possibilitou refletir sobre o desenvolvimento de pensamento crítico no percurso da formação (Riegel, et al., 2021). É notório que o educador tem o papel de facilitador na aprendizagem, atuando na identificação do perfil dos alunos envolvidos nas atividades, bem como utiliza das observações realizadas para o planejamento da aprendizagem. (Fontana, et al., 2020).

Evidencia-se que o processo pedagógico que estabelece a importância de inclusão de atividades de competências relacionadas à pesquisa, auxilia na condução de aprendizagem ao pensamento crítico a partir dos apontamentos já citados (Riegel et al, 2021), mas sobretudo executar tarefas que implementem habilidades cognitivas para pesquisa de campo e de pesquisas na área da educação (Riegel et al., 2018). O pensamento crítico é uma importante competência que deve ser adquirida e aperfeiçoada na formação de profissionais de nível superior (Brasil, 2001).

Aponta-se os caminhos para a construção do pensamento crítico na Enfermagem, assim como torna-se fundamental neste processo que as instituições de ensino promovam um sistema de ensino como foco na formação por competências e pensamento crítico, a fim de que estes sejam instrumentalizados para uma prática profissional que considere a realidade (Riegel et al., 2018). Em continuidade deve-se compreender que as metodologias de ensino são mediadoras e desenvolvem capacidades cognitivas e metacognitivas que devem ser consideradas neste processo (Carbogim, et al., 2017, et al., 2018).

O programa de iniciação científica, que treina estudantes para a pesquisa, tem esse papel e possibilita maturidade intelectual para compreender a ciência e a articulação em seu entorno (Fava-de-Moraes \& Fava, 2000). Para isso, é necessário identificar o nível de conhecimento e criar ações que possibilitem aperfeiçoar as habilidades observadas ou não no discente (Riegel \& Crosseti, 2018).

A partir destes apontamentos, quando da formação de pesquisadores para atuação em pesquisa em contextos de vulnerabilidades, aponta-se que conceitos como de vulnerabilidade (Ayres, 2003) deve ser conferido nas ações, a fim articular com as determinações sociais em saúde (Buss et al., 2007). Logo, a instrumentalização para efetiva prática de pesquisa deve ser conferida no ato do planejamento, e para isso, é necessário construir guias que apresentam elementos equânimes.

Salienta-se que para a construção do instrumento guia, sinalizada no percurso deste relato, partiu-se do princípio que as questões da saúde são inerentes às condições socioambientais e culturais (Campos, 2002), e, portanto, é necessário construir instrumentos de pesquisas que se direcionam para as realidades e suas relações. Ademais, há uma lacuna de referências que consideram as políticas públicas de saúde do Sistema Único de Saúde às populações da Água, Campos e Florestas. 
Neste sentido este relato tem o objetivo demonstrar a construção de um instrumento guia para pesquisa de campo com populações Amazônicas a partir do método Blended Learning, considerando as interações dentro de um Grupo de Estudo e Pesquisa e um projeto de pesquisa da Faculdade de Enfermagem da Universidade Federal do Pará durante o período remoto emergencial.

\section{Metodologia}

\section{Tipo de estudo}

Estudo descritivo do tipo relato de experiência realizado a partir do método Blended Learning (BL), que visa detalhar experiências vivenciadas pelos autores, de modo a expressar um acontecimento (Kich, 2020). O método proposto foi escolhido por associar o ensino remoto com o presencial, desenvolvendo um espaço de formação híbrido, ou seja, a junção de modalidades diferentes de ensino-aprendizagem (Oliveira, et al., 2020) Este método foi potencialmente utilizado dentro das universidades, pois possibilitou a continuidade das atividades acadêmicas em virtude do cenário mundial de 2020 e 2021.

Destaca-se que, a partir de março de 2020 no Brasil, houve mudanças significativas na condução do ensino superior em função da situação sanitária relacionada à pandemia da COVID-19. Pela influência da pandemia causada pelo SARS-CoV2 que gerou adequações na sociedade e a necessidade de isolamento, essa modalidade foi incorporada na instituição em que se desenvolveu a experiência. O BL, que traz a proposta da união do ensino "face-a-face" e as Tecnologias de Informação e Comunicação (TIC) buscando a aprendizagem contínua (Soares \& Martins, 2020) já vinha sendo utilizado em atividades à distância, e com a pandemia estudos têm apontado sua ampla utilização principalmente no ensino (Spinardi, 2018; Silva, et al., 2017; Yaegashi, 2017).

A proposta apresentada pelo BL deve ser conduzida por um professor-facilitador, de modo que este desperte o interesse do aluno em um tema e/ou atividade previamente direcionadas a partir de espaço de troca de ideias, em encontros presenciais e por meio de um fluxo de conhecimento em ambientes virtuais (Pereira, 2020). Tornou-se, portanto, essencial para o direcionamento da construção do instrumento guia, pois a utilização de plataformas e encontros on-line para a troca de informações e criação do material e o momento presencial de aplicação do instrumento é um das articulações inseridas (Pereira, 2020; Soares et al., 2020).

Desenvolveu-se por meio de um treinamento via de Grupo de Estudo e Pesquisas e projeto de pesquisa da Faculdade de Enfermagem da Universidade Federal do Pará, pertencente ao Instituto de Ciências da Saúde, durante o período remoto emergencial entre os meses de março a agosto de 2021, observando as recomendações da resolução $\mathrm{n}^{\circ} 1.513$ de 21 de dezembro de 2020 (Consepe, 2020). Contou com a participação de três discentes e um docente. As atividades foram orientadas e realizadas por meio da plataforma Google Meet e Google Drive, que são ferramentas para a realização de reuniões síncronas (videoconferências) e armazenamento de arquivos, respectivamente, (Melo, 2020) tendo a finalidade de construir o instrumento guia da coleta de dados da pesquisa.

$\mathrm{O}$ treinamento que gerou o instrumento-guia teve como objetivo instrumentalizar discentes de iniciação científica para pesquisas de campo sob orientação de características de cenários socioculturais de povos e populações da Amazônia. Teve como base a comunidade quilombola do Baixo e Médio Itacuruçá, pertencentes ao território das ilhas de Abaetetuba, integrante da $6^{\circ}$ região de saúde do estado do Pará. Para tal foi realizada a busca em bases de dados da Scielo e BVS estudos sobre a região.

O aporte teórico e para análise da experiência fundamentou-se na Teoria da Atividade (TA) (Engestrom, 2010; Leontiev, 2012). A TA possibilita apresentar como as interações realizadas para construção do guia foram importantes para sua efetivação, assim como do construto seres humanos e mídias que deram suporte em virtude da condição sanitária. Ademais, a 
TA também possibilita por meio de uma das suas vertentes compreender os processos de ensino e aprendizagem e os elementos que a compõem, mostrando a dinâmica que constituem a atividade.

\section{Cenário}

Ao longo da vigência do treinamento, que ocorreu no período de agosto de 2020 a fevereiro de 2021 houve a necessidade de algumas adaptações, haja vista que a universidade em dezembro de 2020 criou um Grupo de Trabalho que analisava o cenário epidemiológico do Pará e estabelecia o bandeiramento mais adequado para o período. Esse grupo determinava as recomendações de funcionamento das atividades acadêmicas e administrativas baseadas em estudos científicos e pautadas nas recomendações da Secretaria Especial de Saúde Pública do Pará (SESPA) (Consepe, 2020).

Desse modo, a condução de pesquisas de campo e a entrada de pesquisadores em cenários elencados para coleta de dados de pesquisas foi orientada por meio das determinações deste bandeiramento. Reitera-se que desde abril de 2021 o bandeiramento estava vermelho e as atividades presenciais da pesquisa estavam suspensas, para posteriormente seguir em abril para laranja representando a realização de atividades presenciais mediante justificativa e em maio e junho o bandeiramento encontrava-se no amarelo que permitia o retorno presencial respeitando as orientações de biossegurança e lotação máxima de $30 \%$ dos espaços, incluindo a liberação de pesquisas em campo (Consepe, 2020; Consepe, 2021).

Em continuidade, em virtude do início da campanha de vacinação contra COVID-19 e ao esquema vacinal ter sido oferecido à totalidade dos estudantes, inicia-se a vigência da bandeira verde que permite o retorno das atividades acadêmicas presenciais em todos os cursos com lotação máxima de 50\% dos ambientes, comprovação da vacinação e o uso de máscaras. Diante do exposto, as atividades online aconteceram em março, abril e maio para o aprofundamento na temática e construção do material, e em junho aconteceu a atividade presencial com a aplicação dos instrumentos em um estudo piloto para posterior adequação e coleta final (Consepe, 2021).

Por se tratar de um relato de experiência dos autores, não há necessidade de aprovação pelo CEP/CONEP, ademais o relato inseriu-se no item VIII da resolução 510/2016 enquanto atividade executada exclusivamente para a educação, ensino ou treinamento.

\section{Resultados}

Para a realização da experiência foi realizado o registro do plano de trabalho junto a Pró-reitoria de Pesquisa (PROPESP), o que condicionou a execução das atividades elencadas no cronograma. A Etapa 1 que previa a organização do plano de trabalho e levantamento bibliográfico pôde ser executada sem prejuízo da condição pandêmica. Foi considerado inicialmente o segundo semestre de 2020. Como seguimento e conforme Quadro 1 o planejamento do Grupo de Trabalho elencou as atividades que seriam realizadas durante os seis meses de duração do projeto, ocorrendo a explanação das etapas do plano, a busca da autorização do representante da comunidade quilombola para posterior submissão ao comitê de ética.

Em paralelo, foi desenvolvido um treinamento em plataformas online que tem relação direta com pesquisa, como por exemplo a Plataforma Brasil. Essa plataforma é uma ferramenta nacional e unificada de registros de pesquisas envolvendo seres humanos que permite o acompanhamento dos estudos, bem como disponibiliza à sociedade o acesso aos dados públicos das pesquisas aprovadas (Brasil, 2021). Neste momento o método BL já estava sendo executado, haja vista que permitia dar continuidade às atividades elencadas.

Em um segundo momento, ainda na Etapa 1, foi realizada uma pesquisa bibliográfica a partir de descritores: modo de vida, populações vulneráveis, grupos étnicos; Amazônia e população rural, no LILACS, SCIELO, BDENF E MEDLINE coma combinação do termo booleano "AND". A partir deste levantamento foram solicitadas leituras sobre os artigos encontrados a fim de direcionar o entendimento dos conceitos necessários para a construção dos instrumentos, como observado no Quadro 2. 
As leituras direcionaram os seguintes elementos e conceitos: DSS, sociedade, região, ambiência, território e políticas públicas de saúde.

A próxima etapa consistiu na criação de um quadro que abrange-se os DSS presentes em uma comunidade quilombola da Amazônia tendo como referencial teórico Dahlgren e Whitehead (1991), que demonstra em camadas os fatores relacionados a vida das pessoas, onde os indivíduos estão na base do modelo, com suas características biológicas (idade, sexo, fatores genéticos), logo depois vem o Estilo de Vida, as Redes Sociais Comunitárias, as Condições de Vida e Trabalho e, por fim, as Condições Socioeconômicas e Culturais. Com essa base teórica cada discente produziu um guia e compartilhou com os demais, por meio de encontros online e em grupo durou durante três semanas, possibilitando troca de conhecimento e aperfeiçoamento desse instrumento (Melo, 2020).

Quadro 1 - Demonstrativo das atividades de treinamento executadas pelo grupo.

\begin{tabular}{|c|c|c|c|c|}
\hline ESTRATÉGIA & APLICAÇÃO & $\begin{array}{c}\text { REFERÊNCIA } \\
\text { BIBLIOGRÁFICA }\end{array}$ & $\begin{array}{c}\text { PARTICIPAÇÃO } \\
\text { DO ALUNO }\end{array}$ & RESULTADO ESPERADO \\
\hline $\begin{array}{l}\text { Orientação dos discentes } \\
\text { para buscar embasamento } \\
\text { científico para a } \\
\text { compreensão do assunto. }\end{array}$ & $\begin{array}{l}\text { Estudo sobre o tema } \\
\text { a partir de pesquisa } \\
\text { bibliográfica }\end{array}$ & $\begin{array}{l}\text { Yin, } 2015 \\
\text { Gil, } 2021\end{array}$ & $\begin{array}{l}\text { Buscar nas bases de } \\
\text { dados estudos que } \\
\text { remetem ao tema e } \\
\text { armazená-los para } \\
\text { posterior consulta. }\end{array}$ & $\begin{array}{l}\text { Seleção dos artigos relacionados à } \\
\text { temática e conhecimento } \\
\text { aprofundado pelo discente. }\end{array}$ \\
\hline $\begin{array}{l}\text { Compreensão dos DSS } \\
\text { inseridos no contexto de } \\
\text { comunidades da Amazônia }\end{array}$ & $\begin{array}{l}\text { Montagem do } \\
\text { quadro com pontos } \\
\text { das DSS }\end{array}$ & $\begin{array}{l}\text { Dahlgren } \\
\text { \&Whitehead, } 1991 \\
\text { Buss, } 2007\end{array}$ & $\begin{array}{l}\text { Com base na } \\
\text { literatura científica } \\
\text { elaborou o checklist } \\
\text { com os DSS }\end{array}$ & $\begin{array}{l}\text { Criação de um quadro contendo } \\
\text { um domínio e pergunta norteadora } \\
\text { para a verificação em forma de } \\
\text { checklist sobre os DSS. }\end{array}$ \\
\hline $\begin{array}{l}\text { Cooperação dos discentes e } \\
\text { da docente para a avaliação } \\
\text { dos instrumentos } \\
\text { elaborados. }\end{array}$ & $\begin{array}{l}\text { Compartilhamento } \\
\text { dos resultados dos } \\
\text { primeiros quadros e } \\
\text { figuras }\end{array}$ & $\begin{array}{l}\text { Creswell, } 2010 \\
\text { Bardin, } 2016\end{array}$ & $\begin{array}{l}\text { Apresentação dos } \\
\text { quadros e figuras } \\
\text { construídas e sua } \\
\text { fundamentação } \\
\text { científica. }\end{array}$ & $\begin{array}{l}\text { Exposição e discussão dos quadros } \\
\text { e figuras elaborados pelos } \\
\text { discentes. }\end{array}$ \\
\hline $\begin{array}{l}\text { Aplicação do guia em uma } \\
\text { comunidade a fim de } \\
\text { verificar se estão adequados } \\
\text { ou se necessitam de } \\
\text { adaptações. }\end{array}$ & Estudo piloto & $\begin{array}{l}\text { Zaccaron, et al., } \\
2018\end{array}$ & $\begin{array}{l}\text { Participar da } \\
\text { aplicação dos } \\
\text { instrumentos no } \\
\text { estudo piloto e } \\
\text { analisar possíveis } \\
\text { correções. }\end{array}$ & $\begin{array}{l}\text { Verificação da funcionalidade dos } \\
\text { instrumentos de coleta de dados. }\end{array}$ \\
\hline
\end{tabular}

Fonte: Autores (2022).

A construção metodológica pautou-se em três elementos constitutivos que representam a síntese do que deveria estar presentes no instrumento-guia: Conceitos, Articulações com Populações Amazônicas e referências utilizadas conforme Quadro 2. E após as devidas correções pelo professor-orientador e adaptações necessárias, a versão preliminar foi definida conforme Quadro 3. 
Quadro 2: Quadro síntese para construção do instrumento guia para pesquisa de campo, 2022

\begin{tabular}{|c|c|c|}
\hline $\begin{array}{c}\text { ARTICULAÇÕES COM AS } \\
\text { POPULAÇÕES AMAZÔNICAS }\end{array}$ & CONCEITOS & $\begin{array}{l}\text { REFERENCIAIS } \\
\text { TEÓRICOS }\end{array}$ \\
\hline DSS & $\begin{array}{c}\text { Ambiente, trabalho, saúde, estratificação socioeconômica e } \\
\text { redes de apoio. }\end{array}$ & $\begin{array}{l}\text { Dahlgren e Whitehead } \\
\qquad(1991)\end{array}$ \\
\hline SOCIEDADE & $\begin{array}{c}\text { Conjunto das instituições e a } \\
\text { cultura de um grupo de pessoas distintas, em que seus } \\
\text { comportamentos são afetados por } \\
\text { normas e valores compartilhados. }\end{array}$ & Silva (2008) \\
\hline REGIÃO & Identificação de área delimitada geograficamente & Gomes (1995) \\
\hline AMBIÊNCIA & $\begin{array}{l}\text { O ambiente de vivência das pessoas que possuem condições } \\
\text { físicas e emocionais que geram bem-estar }\end{array}$ & Bestetti (2014) \\
\hline TERRITÓRIO & $\begin{array}{l}\text { Espaço social de controle político-econômico resultante da } \\
\text { apropriação simbólica pela vivência de um grupo }\end{array}$ & Haesbaert (2002, p.121) \\
\hline POLÍTICAS PÚBLICAS DE SAÚDE & $\begin{array}{l}\text { Ações e programas governamentais que visam melhorar as } \\
\text { condições de saúde }\end{array}$ & Opas (2004) \\
\hline
\end{tabular}

Fonte: Autores (2022).

Quadro 3: Instrumento guia, 2022.

\begin{tabular}{|c|c|c|c|}
\hline Domínio & Pergunta & SIM & NÃO \\
\hline \multirow{2}{*}{ Organização do registro de campo } & Comunicar representante da ARQUIA sobre as datas do registro do campo & & \\
\hline & Solicitar permissão do registro fotográfico para os sujeitos da pesquisa & & \\
\hline \multirow{5}{*}{ Ambiente } & Possui rede de esgoto? & & \\
\hline & Possui água encanada? & & \\
\hline & Possui risco de contaminação hídrica? & & \\
\hline & Possui risco de contaminação do solo? & & \\
\hline & Utilizam dos recursos naturais para sua sobrevivência? & & \\
\hline \multirow{4}{*}{ Trabalho } & As pessoas precisam se deslocar para o trabalho? & & \\
\hline & Possuem trabalho na própria comunidade como fonte de renda? & & \\
\hline & O trabalho exercido dentro da comunidade apresenta algum risco ocupacional? & & \\
\hline & Existe incentivo de cursos profissionalizantes na comunidade? & & \\
\hline \multirow{2}{*}{ Saúde } & Possui doenças decorrentes da falta de saneamento? & & \\
\hline & Possui algum serviço de saúde na comunidade? & & \\
\hline
\end{tabular}




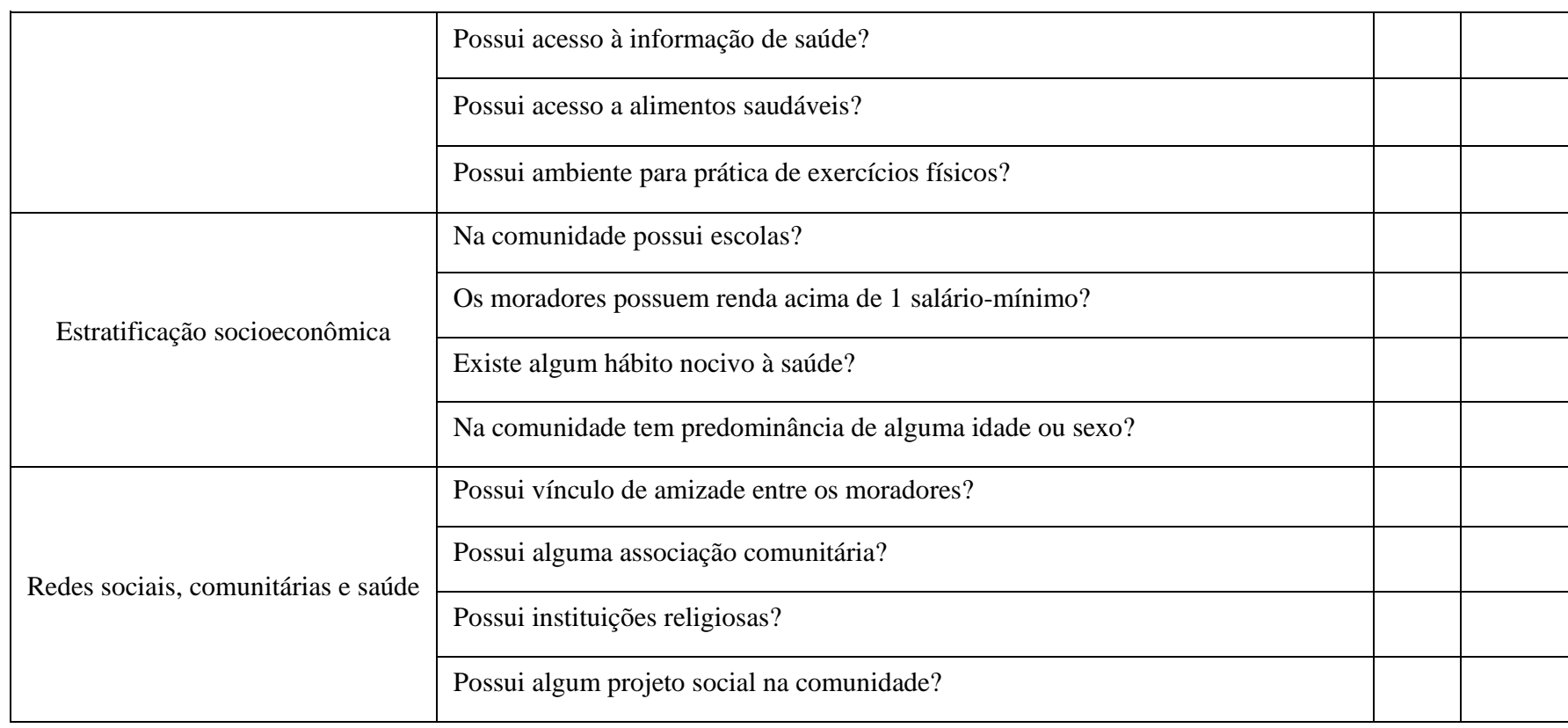

Fonte: Baseado nos Determinantes Sociais de Saúde dos autores Dahlgren e Whitehead (1991).

Com o instrumento-guia finalizado considerou-se a aplicação do guia em um estudo piloto para testar a adequação do instrumento à realidade do local, sendo possível realizar as adaptações que se julguem necessárias para a coleta de dados definitiva. A entrada na comunidade aconteceu via rodo-fluvial e rodoviária, acompanhado do instrumento guia e de um diário de campo, percebeu-se que a etapa de levantamento bibliográfico foi fundamental para a compreensão do modo de vida encontrado na comunidade.

Foi respeitada a condição sanitária vigente ao entrar nos espaços públicos. Como proposta o guia tinha seguimento socioambiental, e, portanto, não houve nenhuma interlocução com moradores da região neste momento, pois o foco foi o ambiente. Todos os registros e anotações seguiram os apontamentos do guia, sendo apenas utilizado um diário de campo para suporte e anotações das observações pertinentes e para futuras adequações do instrumento-guia.

\section{Discussão}

\section{Implementação das atividades de iniciação científica}

A Iniciação Científica (IC) é considerada como componente imprescindível no Ensino Superior, pois aproxima o aluno do campo de pesquisa, permitindo o desenvolvimento de pensamentos críticos diante da realidade estudada e encoraja o estudante pesquisador na resolução de novos problemas, assim como um modo especial de despertar a vocação científica de graduandos, estimulando-os à pós-graduação (Luz, et al., 2020). Nota-se que a IC permite que a aprendizagem seja movida por relações em âmbito social e mediada por uma ação, assim como das relações promovidas durante sua execução e de um objetivo motivador como prevê a TA (Leontiev, 2012). Torna-se então uma ferramenta essencial para motivar alunos para alcançar um objetivo.

Embora muitos desafios perpassem a atuação dos pesquisadores das ciências da saúde, é importante ressaltar o quanto a IC contribui na formação acadêmica do aluno da área da saúde. Para Pirola e colaboradores (2020), a IC constitui-se como uma atividade fundamental no processo de formação/aprendizado na graduação, pois possibilita descobertas significativas no campo da saúde, entendimento acerca do modo de vida e especificidades de comunidades tradicionais brasileiras e constatação de evidências na realidade em saúde no Brasil. Assim, com o atual contexto pandêmico, evocou-se dos estudantes de enfermagem a necessidade de adquirir novos conhecimentos, habilidades técnico-científicas que demonstram a capacidade de 
investigar, diagnosticar, estabelecer metas e intervir com base em evidências e pensamento crítico novas metodologias no que se refere a ao aprendizado da enfermagem (Riegel, et al., 2021).

As ferramentas tecnológicas educacionais popularizaram-se ainda mais com a chegada da COVID-19, tão logo, as atividades das instituições de ensino, outrora presenciais, ajustaram-se à forma on-line - o denominado Estudo Remoto Emergencial (ERE). Desse modo, destaca-se que por meio da IC habilidades são implementadas no processo de formação do aluno como identificado pelo método BL. Diante disso, conforme visualizado no Quadro 1, as atividades de IC não cessaram, mas adaptou-se por meio de atividades on-line.

É possível visualizar a realização de leituras bibliográficas prévias, encontros para discussões, transmissão de informações acerca do objeto de estudo e delimitação organizacional na construção do instrumento de pesquisa, o que possibilita observar que ocorre sendo guiada por ações e operações a fim de alcançar um objetivo (Leontiev, 2012 ). Neste sentido, emergiram desafios quanto à exploração da temática e do seu entendimento de forma minudente. Posto isso, sinalizase a importância da organização do processo metodológico da pesquisa, bem como o papel de destaque do professor orientador, que segundo Melo (2021) possui papel relevante no desenvolvimento intelectual e humano do aluno assegurando êxito em seu processo de aprendizagem.

Em virtude da necessidade do distanciamento social e das recomendações sanitárias durante o ERE, as pesquisas de IC ficaram limitadas, fazendo com que o pesquisador segue à risca os protocolos de saúde recomendados e delimitaram as idas ao campo de pesquisa (Oliveira, 2021). Percebeu-se que houve limitações para entrada em territórios elencados para pesquisa, o que obrigou que estratégias fossem realizadas no sentido de manter a formação de pesquisadores. De acordo com Sena et al., (1999) e Riegel, et al., (2021), as metodologias ativas emergidas durante o ERE permitem o fortalecimento de novas bases de aprendizado mediadas pela tecnologia, buscando formas de organizar o ensino e pesquisa, e assim explorar habilidades e competências dos estudantes pesquisadores capazes de subsidiar o cuidado humano, éticos, seguros e baseados em evidências científicas assegurando o pensamento crítico fundamentais à formação acadêmica.

No percurso do planejamento metodologia de trabalho conforme Quadro 1 as estratégias foram implementadas e orientadas para promover o aprendizado contínuo sobre conceitos, suas aplicabilidades e instigar no aluno a prática de pesquisa bibliográfica. Para Teixeira, et al., (2020) e Yin (2015), o levantamento de dados bibliográficos é feito a partir do estudo em torno de documentos que servirão como um planejamento estratégico, e para embasamento e compreensão do assunto que se deseja estudar. Nota-se com isso que a participação dos alunos no percurso do planejamento organizacional do seu projeto de pesquisa, é indispensável para gerar credibilidade e confiabilidade nos resultados esperados e alcançados e contribui substancialmente como ponto de referências para estudos posteriores e servir de dados para futuras pesquisas (Ferreira, et al., 2018).

\section{A formação de pesquisadores para pesquisa no contexto amazônico e Instrumentos que consideram a realidade regional}

O saber do pesquisador configura, a partir de um preparo temporal, fundamentação teórica e a expertise frente às especificidades do meio. No contexto amazônico, as demandas dos serviços no contexto cultural, social e ambiental mostram a necessidade e a importância do discernimento do pesquisador para a diversidade e a complexidade com as peculiaridades do estudo diante das características amazônicas. (Dias, et al., 2020). As atividades de treinamento para formação de pesquisadores devem considerar esse plano social e os aportes teóricos são fundamentais para entender o propósito da atividade (Leontiev, 2012).

Dentre aspectos importantes, a fundamentação conceitual pertencentes e pertinentes, observado no Quadro 2, os conceitos fundamentados para os Determinantes Sociais de Saúde (Dahlgren e Whitehead, 1991), Sociedade (Silva, 2008), 
Região (Gomes, 1995), Ambiência (Bestetti, 2014), Território (Haesbaert, 2002) e Políticas Públicas de Saúde (OPAS,2004) agrega a complexidade e fomenta a compreensão do espaço metodológico científico, como também no olhar crítico-reflexivoinvestigativo, além de propiciar o encadeamento de um método mais organizado e direcionado. (Thomas, et al., 2018), como observado no Quadro 3. Neste momento é dado o suporte teórico para os elementos da atividade e para chegar no objetivo proposto (Leontiev, 2012).

Pontua-se, conforme apontamentos de Bardin (2015), que o trabalho científico, se baseia no desdobramento dos processos lógicos do pensamento e do conhecimento, isto corrobora para formulação de um instrumentos compatíveis e determinante na condução da revelação da problemática. Logo, a compreensão e a extensão dos conceitos, que promovem a elucidação dos significados e os eventos integrando o sujeito, objeto e o cenário de estudo dentro do pensamento crítico, bem como uma elaboração de instrumento de pesquisa. Percebe-se estes apontamentos nas perguntas do Quadro 3, pois a interrogação dirigida para cada domínio conceitual usado, estruturou o desenvolvimento e a observação do objeto de estudo.

Outrossim, a categorização de pontos específicos, como legislações, geográficos-históricos e a antropologia, correlacionados com a conceituação ressaltam o direcionamento de achados importantes na condução e produção de instrumentos de pesquisa. A exemplo, as políticas específicas do lócus de estudo, como Política Nacional de Saúde Integral das Populações do Campo, da Floresta e das Águas (PNSIPCFA) que fomenta o desenvolvimento de projetos de pesquisa e de extensão relacionados à saúde das populações relacionadas aos riscos e qualidade de vida e saúde ancorados com olhares interdisciplinares e éticos nas questões sociais-étnicos rodeiam os envolvidos.

Dessa maneira, a construção de instrumentos nas publicações brasileiras abarca as dimensões: intelectual, sociológica, psicológica e filosófica para a observação, obtenção e a análise das informações coletadas, conduzem ao enfoque multidimensional e holístico, principalmente nos estudos de análise descritiva-qualitativa e de conteúdo (Reigel \& Crossett, 2018). Tal fenômeno, provoca a operacionalização dos conceitos e das variáveis possibilitam a identificação e captação, ressaltando o aprofundamento das questões dos componentes do universo pesquisado não-mensuráveis e subjetivos. (Gil, 2009)

Com isso, o instrumento respaldado em conceitos, produz fragmentos definidores, que apresentam as questões objetivas e mensuráveis, em cada domínio conceitual do trabalho, seguindo a compreensão para a caracterização, articulação e inserção de elementos definidores nas variáveis conceituais. Compreende-se que a visualização desses componentes dentro do pesquisado, resulta na compreensão interligada e simultânea de achados básicos e estruturantes, principalmente em cenários distintos e indissociáveis nas relações do local. (Bardin, 2016; Creswell, 2020) e deste modo conseguimos traçar um instrumento que tenha elementos do objetivo da atividade (Leontiev, 2012 ).

\section{Importância da construção e teste piloto}

Para o desenvolvimento dessas atividades é exigido tempo e dedicação do aluno para a construção do planejamento, nesse período ocorre a formulação de instrumentos claros e funcionais (Thomas, et al., 2018). Buscou-se elaborar os instrumentos de acordo com o objetivo da pesquisa e que tivesse elementos estruturais de fácil interpretação e orientados por conceitos, por isso optou-se pelo formato de checklist que é uma lista de itens a serem verificados, dessa forma diminuindo a incidência de erros e garantindo que todas as etapas sejam devidamente analisadas, tendo o cuidado de não utilizar de não usar fonte muito pequena, ou projetar um instrumento demasiadamente longo (Murilo, 2021).

Outro aspecto importante refere-se a escolha de um referencial teórico que embasasse o processo da pesquisa, haja vista que era necessário construir uma ferramenta que avaliasse de forma abrangente a diversidade que é a população quilombola e ribeirinha da Amazônia. Por conta disso, optou-se pelos apontamentos das DSS que demonstra como as formas de viver de diferentes grupos produzem desgastes e fortalecimentos para a saúde (Buss, 2007) e a antropologia de Malinowski 
que busca compreender a organização social de determinado local com a imersão nos dados e no processo de viver das pessoas (Malinowski, 1978; Marques, 2016).

Para a validação do instrumento, realizou-se o teste piloto para verificar se o guia era eficaz e eficiente. Visto que, uma ferramenta tendenciosa que não é testada, poderá coletar informações imprecisas, distorcendo os possíveis resultados reais da pesquisa (Thwaites, 2016). Desse modo, quaisquer incertezas e dúvidas devem ser esclarecidas até que o instrumento se torne de fácil compreensão e alcance o objetivo da pesquisa (Thomas, et al., 2018).

Sabe-se que o instrumento é válido quando tem as características de ser confiável e preciso (confiabilidade), ser apropriado ao problema de pesquisa (validade de conteúdo), refletir a teoria a ser analisada (validade de construto), e ser capaz de medir as mudanças, tanto em indivíduos diferentes quanto nas respostas de um único indivíduo ao longo do tempo (sensibilidade a alterações) (Yébenes, et al., 2009). Onde o guia construído ao passar pelo teste piloto, mostrou-se viável aos pesquisadores ao abranger essas características e se aproximar das particularidades do local.

O guia mostrou-se eficaz, pois o campo do domínio abrangeu os itens primordiais para verificar o modo de vida da população quilombola. O domínio organização do registro do campo, observou o aceite do representante da comunidade para torna legal a entrada dos pesquisadores, item do ambiente analisou as condições sanitárias, os riscos biológicos para os moradores e a relação deles com o ambiente que o cerca, sendo relevado a ligação dos moradores com a natureza, onde mostrase necessária como uma fonte de renda, lazer e o meio de locomoção (Nascimento, et al., 2021; Pojo et al., 2019).

No quesito trabalho, buscou-se observar o processo de trabalho e as relações observadas a partir da realidade comunidade. Em relação à saúde foi analisado a acessibilidade dos moradores a fatores que repercutem na promoção da saúde de forma integral, a estratificação socioeconômica verificado o acesso à educação e o perfil dos moradores da região e as redes sociais que identificaram as formas de amparo dessas pessoas, seja pelo meio da família, religiosos, associação comunitária, profissionais da saúde, entre outros. Diante dessa perspectiva, o guia conseguiu atingir o objetivo da pesquisa. Nota-se que o guia além de permitir identificar características socioambientais também possibilitou que no percurso do teste piloto as interações do discente de IC com dinâmicas sociais fosse efetivada, o que nos permite observar que as relações se ampliam para além dos espaços das tecnologias digitais usadas para execução das atividades, proporcionando interações sociais e com o meio (Ergestrom, 2010, Leontiev, 2012).

\section{Limitações}

Entende-se que este instrumento não atende necessidades quanto a povos indígenas, ainda que estes possuam características de populações da floresta. É necessário em uma segunda etapa apresentar o instrumento a juízes expertises da área a fim de validar seu conteúdo.

\section{Contribuições}

A experiência contribuiu para realizar reflexões sobre determinações sociais em saúde por meio da construção de instrumento que utilizou arcabouço teórico sobre os problemas e políticas de saúde para as populações em situação de vulnerabilidade. Outrora, possibilitou entender que quando de situações históricas e epidemiológicas como da pandemia, é possível continuar com as atividades de iniciação científica por meio de treinamentos pontuais que possibilitem a construção do pensamento crítico. também possibilita observar relações sociais e com tecnologias e as interações que podem ser construídas. 


\section{Considerações Finais}

O instrumento guia mostrou-se eficaz em direcionar a observação do pesquisador para os determinantes de cada domínio, entretanto percebe-se que para explorar esses domínios era necessário complementar as informações no diário de campo e alinhar com os registros fotográficos. Possibilitou reflexão crítica sobre as condições de vida e trabalho (transportes, alimentação, meios de trabalho, educação, saneamento básico, habitação, segurança e lazer), acessibilidade e acesso à saúde, redes sociais e comunitárias (religiosidade e assistência social) dessa população tradicional.

Para além disso, esta experiência de iniciação científica com método BL proporcionou crescimento profissional dos discentes, pois assumiram uma posição de protagonistas para aquisição de conhecimento e elaboração do instrumento guia, e o professor como facilitador dessa experiência conseguiu desenvolver esse método de forma a ser o mediador do conhecimento. Tal condição demonstrou uma forma de educação transformadora que considerou a realidade dos alunos para a construção gradual de aquisição de competências para a construção dessa ferramenta.

Sugere-se para estudos futuros a necessidade de criar instrumentos específicos para cada grupo étnico e políticas específicas considerando suas especificidades culturais e regionais. Entende-se também que é oportuno desenvolver novos estudos que mostram os registros de campo a partir de narrativas do pesquisador de modo a demonstrar a etnografia pela perspectiva da saúde coletiva.

\section{Agradecimentos}

Programa de Apoio ao Doutor Pesquisador - PRODOUTOR - Universidade Federal do Pará

\section{Referências}

Alves, S. M. \& Teo, C. R. P. A. (2020). O ativo das metodologias ativas: contribuições da teoria histórico-cultural para os processos de ensinar e aprender na educação superior. Educação em revista. 36, e229610. doi: https://doi.org/10.1590/0102-4698229619

Ayres, J. R. de C. M., Franca Junior, I., Calazans, G. J., \& Saletti Filho, H. C. (2003). O conceito de vulnerabilidade e as práticas de saúde: novas perspectivas e desafios. In Promoção da saúde: conceitos, reflexões, tendências ( pp 121-142). Rio de Janeiro, RJ: FIOCRUZ.

Bardin, L. (2016). Análise de conteúdo: edição revista e ampliada. São Paulo, SP: Edições 70.

Bestetti, M. L. T. (2014). Ambiência: espaço físico e comportamento. Revista Brasileira Geriatria e Gerontologia, 17(3), 601-610. doi: http://dx.doi.org/10.1590/1809-9823.2014.13083.

Bordenave, J. D., Pereira, A. M. (1994). Estratégias de ensino-aprendizagem (25a ed). Petrópolis, RJ: Vozes.

Brasil. (2001). Conselho Nacional de Educação. Resolução CNE/CES n 3, de 7 de novembro de 2001. Institui as Diretrizes Curriculares Nacionais do Curso de Graduação em Enfermagem. Diário Oficial da União, Brasília, 9 de novembro de 2001. Seção 1, p. 37.

Brasil. (2021). Sobre a Plataforma +Brasil. https://www.gov.br/economia/pt-br/assuntos/plataforma-mais-brasil/sobre-a-plataforma-mais-brasil/sobre-aplataforma.

Buss, P. M., \& Pellegrini-Filho, A. (2007). Saúde e seus Determinantes Sociais. PHYSIS: Rev. Saúde Coletiva, Rio de Janeiro, 17(1), 77-93. doi: https://doi.org/10.1590/S0103-73312007000100006.

Campos, G. W. de. S. (2002). Sete considerações sobre saúde e cultura. Saúde e sociedade, 11, 105-115. doi: 10.1590/S0104-12902002000100011.

Consepe. (2021a). RESOLUÇÃO N. 5.453, DE 14 DE DEZEMBRO DE 2021. Estabelece o retorno pleno das atividades acadêmicas presenciais e revoga a Resolução $n^{\circ}$ 5.294. Recuperado em disponibilizada em 21 de agosto de 2020, em: https://coronavirus.ufpa.br/1/recomenda\%C3\%A7\%C3\%B5es.

Consepe. (2021b). RESOLUÇÃO N. 1.526, DE 06 DE SETEMBRO DE 2021. Atualiza normas e procedimentos para o trabalho acadêmico e administrativo remoto e/ou presencial durante o período de emergência em saúde pública causada pelo novo Coronavírus e revoga as Resoluções $\mathrm{n}^{\circ} 1.513$, de 21 de dezembro de 2020, e n. 1.524 https://coronavirus.ufpa.br/1/recomenda\%C3\%A7\%C3\%B5es.

Creswell, J. W. (2010). Projeto de pesquisa: métodos qualitativo, quantitativo e misto. Tradução Magda Lopes. 3 ed. Porto Alegre, RS: Artmed. doi: https://doi.org/10.26512/les.v13i1.11610.

Gil, A. C. Métodos e técnicas de pesquisa social. 7 ed. São Paulo, SP: Atlas. 2021.

Dahlgren, G., Whitehead, M. (1991). Policies and Strategies to Promote Social Equity in Health Stockholm. Institute for Future Studies. https://www.iffs.se/media/1326/20080109110739filmz8uvqv2wqfshmrf6cut.pdf 
Engeström, Y. \& Toiviainen, H. (2010). Co-configurational design of learning instrumentalities: An activity-theoretical perspective. Routledge. ISBN 9780203847817

Ferreira, C. L., Schwarzbach, L. C., \& Ferreira, V. C. R. (2018). Instrumento para a coleta de dados primários para pesquisas em administração (número especial). ENACILLA, 1 (spe), 27-33. http://periodicos.unespar.edu.br/index.php/raei/article/view/3275/2153

Flavio, F. D. M., \& Fava, M. (2000). A iniciação científica: muitas vantagens e poucos riscos. São Paulo em perspectiva, 14 (1), $73-77$. doi: https://doi.org/10.1590/S0102-88392000000100008

Fontana, R. T., Wachekowski, G., \& Barbosa, S. S. N. (2020). As metodologias usadas no ensino de enfermagem: com a palavra, os estudantes. Educação em Revista, 36, e220371. doi: https://doi.org/10.1590/0102-4698220371

Gomes, P. C. da C. (1997). O conceito de região e sua discussão. In Castro, I. E. Gomes, P. C. C \& Corrêa, R. L. [Orgs.]. Geografia: conceitos e temas (pp 4976). Rio de Janeiro, RJ: Bertrand Brasil

Haesbaert, R. (2002). Territórios alternativos. Niterói: EdUFF; São Paulo: Contexto

Kich, J. I. D. F. (2020). Blended Learning na Prática: O Caso do Curso Superior de Administração do Centro Universitário Estácio de Santa Catarina. Revista Internacional Educação. Superior, 6, 1-28. e020014. doi: https://doi.org/10.20396/riesup.v6i0.8654971

Leontiev, A. N. (2012). Uma contribuição à Teoria do Desenvolvimento do Psique Infantil. In: Vitgotki, L.S. Luria AR. Leontiev AN [Orgs]. Linguagem, desenvolvimento e aprendizagem (pp 59-83). São Paulo, SP: Ícone

Luz, B. da., Teixeira, J., Santos, R. P. G. dos, \& Bitencourt, S. P. (2020). A formação humana na iniciação científica: a pandemia e os dilemas históricos educativos. In Anais do XIX Encontro de História da Anpuh-Rio sobre história do futuro: ensino, pesquisa e divulgação científica. Rio de Janeiro, RJ: AnpuhRIO. https://www.encontro2020.rj.anpuh.org/anais/trabalhos/trabalhosaprovados

Malinowski, B. (1978). Argonautas do Pacífico Ocidental. São Paulo, SP: Abril Cultural

Marques, J.P. (2016). A “observação participante" na pesquisa de campo em Educação. Educação em Foco, 19 (28), 263-284. doi: https://doi.org/10.24934/eef.v19i28.1221

Melo, F. C. C., Costa, R. F. R., \& Corso, J. M. D. (2020). Modelo conceitual aplicável a estudos sobre determinantes sociais da saúde em municípios brasileiros. Saúde e Sociedade, 29 (2), e181094. ISSN 1984-0470. doi: https://doi.org/10.1590/S0104-12902020181094

Melo, M. M. O. (2021). Projeto de iniciação científica: experiência acadêmica em temática na pandemia. Ensino em Perspectivas, 2(4), 1-6. ISSN: 2675-9144. https://revistas.uece.br/index.php/ensinoemperspectivas/

Melo, W. V. B. (2020). Como utilizar o google Meet. Secretaria de Programas em educação aberta e a distância (SPREAD) da Universidade Federal de Pernambuco

Moran, J. (2017). Metodologias ativas e modelos híbridos na educação In Yaegashi, S.F.R., Bianchini, L. G. B, Júnior Oliveira, I. B. Santos, A.R., \& Silva, S.F.K. (Orgs). Novas Tecnologias Digitais: Reflexões sobre mediação, aprendizagem e desenvolvimento (pp: 23-35). Curitiba: CRV. doi: http://dx.doi.org/10.24824/978854441989.2

Murilo, F. M. S. (2021). Checklist: o que é e como aplicá-lo para quase tudo. https://www.fm2s.com.br/o-que-checklist-saiba-como-aplicar-para-quase-tudo/

Nascimento, M. T. A, Carvalho, J. F. C., \& Castro, N. J. C. (2021). Ir e vir de uma comunidade quilombola em meio a pandemia. AntHropológicas Visual, 7(2). doi: https://doi.org/10.51359/2526-3781.2021.251559

Oliveira, E. E., Freitas, T. C., Sousa, M. R., Mendes, N. C. S. G. M., Almeida, T. R., Dias, L. C.,Ferreira, A. L. M., \& Ferreira, A. P. M. (2020). A educação a distância $(\mathrm{EaD})$ e os novos caminhos da educação após a pandemia ocasionada pela Covid-19. Brazilian Journal of Develop, 6(7), 52860-52867. doi: https://doi.org/10.34117/bjdv6n7-799.

Oliveira, V. H. N. (2021). Desafios para a pesquisa no campo das ciências humanas em tempos de pandemia da COVID-19. Boletim de Conjuntura (BOCA), 5(14), 93-101. doi: http://dx.doi.org/10.5281/zenodo.4513773.

Opas (2004). Políticas públicas em Saúde Pública / Patrícia T. R. Lucchese, coord, Dayse Santos Aguiar,Tatiana Wargas,Luciana Dias de Lima,Rosana Magalhães, Giselle Lavinas Monerat. São Paulo: BIREME/OPAS/OMS.

Pereira, J. A. (2020). O ensino com ênfase na aprendizagem colaborativa - reflexão sobre uma experiência na disciplina de teoria do conhecimento. Educação Por Escrito, 11(2), e30993. doi: https://doi.org/10.15448/2179-8435.2020.2.30993

Pirola, S. B. D. F. B., Padilha, F. D., Di Mauro, J. M. B, Pirola, L. H. F. B., \& Gabriel, S. A. (2020). A importância da Iniciação Científica na graduação de Medicina. Revista Corpus Hippocraticum, 1(1). http://revistas.unilago.edu.br/index.php/revista-medicina/article/view/232

Pojo, E. C., \& Elias, L.D. (2019). O cotidiano das águas na tradição quilombola da comunidade do rio baixo Itacuruçá-Abaetetuba, Pa. Tempos Históricos, 22(2), 49-72. doi: https://doi.org/10.36449/rth.v22i2.20509

Riegel, F., Martini, J. G., Bresolin, P., Mohallem, A. G. C., \& Nes, A. A. G.(2021). Desenvolvendo o pensamento crítico no ensino de Enfermagem: um desafio em tempos de pandemia de Covid-19 (número especial). Escola Anna Nery, 25(spe), e20200476. doi: https://doi.org/10.1590/2177-9465-EAN-20200476

Riegel, F., Crossetti, M. G. O. (2018). Referenciais teóricos e instrumentos para avaliação do pensamento crítico na enfermagem e na educação. Revista Gaúcha de Enfermagem, 39, e2017-0097. ISSN 1983-1447. Doi: https://doi.org/10.1590/1983-1447.2018.2017-0097 
Research, Society and Development, v. 11, n. 2, e45711226124, 2022

(CC BY 4.0) | ISSN 2525-3409 | DOI: http://dx.doi.org/10.33448/rsd-v11i2.26124

Sena, R. R. \& Brant, M. J. C. G. (1999). Iniciativa de inovação do ensino de Enfermagem na América Latina. Revista Brasileira de Enfermagem, 52(2), 243250. doi: https://doi.org/10.1590/S0034-71671999000200011

Silva, E. W. (2008). Sociedade, política e cultura. Ijuí, RS: Universidade Regional do Noroeste do Estado do Rio Grande do Sul. https://bibliodigital.unijui.edu.br:8443/xmlui/bitstream/handle/123456789/244/Sociedade, $\% 20$ p $\%$ C3\%B3litica\%20e\%20cultura.pdf?sequence=1

Silva, M. R. C., Maciel, C., Alonso, K. M. (2017). Hibridização do ensino nos cursos de graduação presenciais das universidades federais: uma análise da regulamentação. RBPAE, 33(1), 95-117. doi: https://doi.org/10.21573/vol33n12017.74042

Soares, J. R. S., \& Martins, J .L. (2020). Blended Learning: Uma Visão Geral sobre o êxito no Ensino Híbrido por meio de Experiências Internacionais. Palmas, Tocantins [Dissertação de mestrado, Universidade Federal de Tocantins, Palmas, TO]. Publicação eletrônica antecipada. doi:http://dx.doi.org/10.13140/RG.2.2.27495.06562

Donato Spinardi, J., \& José Both, I. (2018). Blended learning: o ensino híbrido e a avaliação da aprendizagem no ensino superior. Boletim Técnico Do Senac, 44(1). doi: https://doi.org/10.26849/bts.v44i1.648

Thomas D. B., Oenning N. S. X., \& Goulart B. N. G. (2018). Aspectos essenciais na construção de instrumentos de coleta de dados em pesquisas primárias de saúde. Rev. CEFAC, 20(5):657-664. doi: http://dx.doi.org/10.1590/1982-021620182053218

Thwaites Bee, D., \& Murdoch-Eaton, D. (2016). Questionnaire design: the good, the bad and the pitfalls. Arch Dis Child - Educ Pract Ed,101(4), 210-212. doi:http://dx.doi.org/10.1136/archdischild-2015-309450.

Universidade Federal do Pará (2021). Conselhos Superiores definem o retorno pleno das aulas presenciais, a adoção do passaporte vacinal e novas orientações para o bandeiramento na UFPA. Belém/Pará, Assessoria de Comunicação Institucional da UFPA. https://coronavirus.ufpa.br/not\%C3\%ADcias/conselhossuperiores-definem-o-retorno-pleno-das-aulas-presenciais

Yébenes Prous, M. J. G., Rodríguez Salvanés F., \& Carmona Ortells L. (2009). Validación de cuestionarios. Reumatol Clínica, 5(4):171-177. doi: http://dx.doi.org/ 10.1016/j.reuma.2008.09.007

Yin, R. K. (2015). Estudo de caso:planejamento e métodos. Porto Alegre, RS:Bookman

Zaccaron, R., D’Ely, R. C. de S. F., \& Xhafaj, D. C. P. (2018). Estudo piloto: um processo importante de adaptação e refinamento para uma pesquisa quase experimental em aquisição de L12. Revista Do GELNE, 20(1), 30-41. doi: https://doi.org/10.21680/1517-7874.2018v20n1ID13201 